Rechtsmedizin 2005 - 15:143-147 DOI 10.1007/s00194-005-0318-2

Online publiziert: 29. April 2005

(c) Springer Medizin Verlag 2005

G. Stoppe · Universitäre Psychiatrische Kliniken, Basel

\title{
Die Verhandlungsfähigkeit des alten (multimorbiden) Patienten
}

ausgewiesenen Sachverständigen. Inwieweit hier schon - zumindest für den alten Patienten - standardisierte Hilfsmittel in Form strukturierter ,assessments“ einbezogen werden sollten, wird in diesem Beitrag thematisiert. Im Folgenden wird deshalb der in der medizinischen Literatur (international) häufiger verwendete Begriff der Kompetenz statt des Terminus „Verhandlungsfähigkeit" verwendet.

Gesundheitliche Störungen, die die Kompetenz beeinflussen, sind im Alter vorwiegend aus 3 Gebieten zu erwarten: Demenzerkrankungen, Depressionen und Delirien. Hirnorganische Beeinträchtigungen durch somatische Erkrankungen und die damit zusammenhängende Medikation äußern sich psychopathologisch in der Regel in Form eines dieser Syndrome. Zunächst sollen deshalb diese Krankheitsbilder charakterisiert werden.

\section{Für die Verhandlungsfähigkeit relevante Krankheitsbilder}

\section{Demenzen}

Schaut man sich die gegenwärtig verwendete Operationalisierung eines Demenzsyndroms an, wie sie sowohl die „International Classification of Diseases (ICD-)10“ [25] als auch das „Diagnostic and Statistical Manual of Mental Disorders (DSM-) IV “ $^{2}$ 2] vorsehen (zusammengefasst in - Abb. 1), so ergibt sich schwerelos, dass die Diagnose einer Demenz immer eine Beeinträchtigung der Kompetenz bedingt. Ein Ermessensspielraum im Sinne eines dann situativ zu bestimmenden "Graubereiches" besteht sicher bei sehr leichten Demenzen $u$ und einfachen Verhandlungsinhalten.

Demenzkrankheiten betreffen in Deutschland derzeit etwa 1 Mio. Menschen, d. h. 6-8\% der Über-65-Jährigen. Der exponenzielle Anstieg sowohl der Prävalenz als auch der Inzidenz bringt es mit sich, dass mit zunehmendem Alter eines Verhandlungsteilnehmers die A-priori-Wahrscheinlichkeit, dass dieser an einer Demenz leidet, ansteigt. Während bei den 6o- bis 64-Jährigen „nur“ $1 \%$ der Bevölkerung betroffen sind, sind dies bei den Über90-Jährigen bereits $35 \%$. Hauptursache für die Erkrankung ist in etwa zwei Dritteln der Fälle die Alzheimer-Krankheit, gefolgt von einem tendenziell eher rückläufigen Anteil vaskulärer Demenzen und Mischformen aus beiden [5]. Vor allen Dingen die Alzheimer-Erkrankung kann heute immer früher und mit hoher diagnostischer Sicherheit (ca. 90\%) bereits vor dem Tod diagnostiziert werden. Hierzu wird auf entsprechende Übersichtsliteratur verwiesen $[23,24]$. Ein wesentliches neurobiologisches Merkmal, das auch besonders mit den kognitiven Einbußen stark korreliert, ist das cholinerge Defizit. Ein cholinerger Zellfunktionsverlust von mehr als 40\% führt zu klinischen Beeinträchtigungen. Diese betreffen nicht nur Lernen und Ge-

Nach einem Vortrag bei der Herbsttagung der Arbeitsgemeinschaft Forensische Psychopathologie und Ethik der Deutschen Gesellschaft für Rechtsmedizin am 26.10.2002 in Göttingen. 


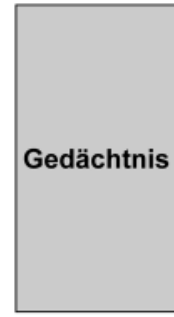

obligat

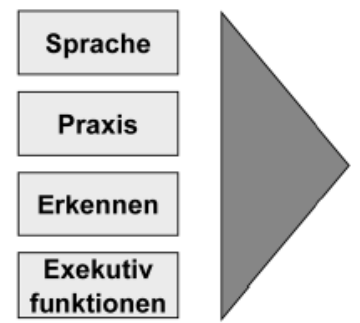

$>1$ Bereich

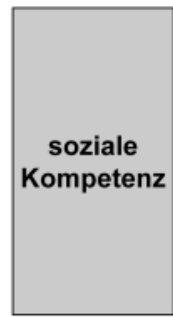

obligat

Abb. $1 \Delta$ Schematische Darstellung der Kriterien zur Diagnose einer Demenz nach dem diagnostisch-statistischen Manual (DSM-IV [2]). Die Kriterien der ICD-10 [25]) sind ähnlich

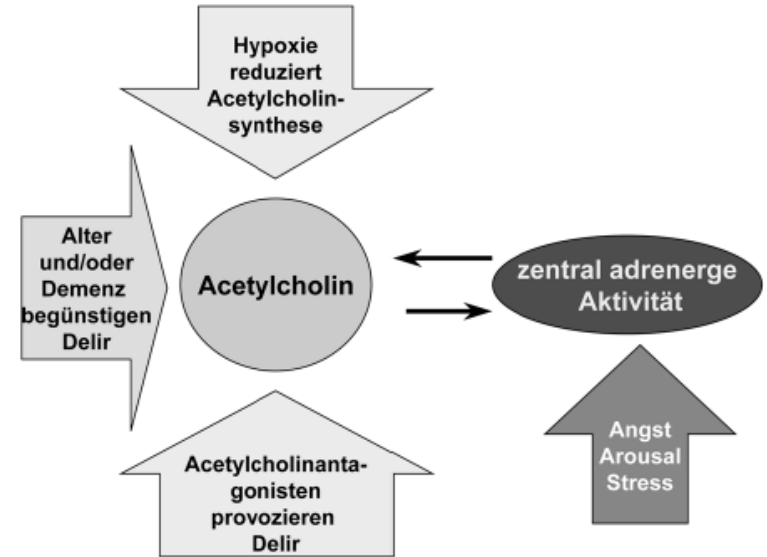

Abb. $\triangle$ Schematische Zusammenfassung der Pathophysiologie eines deliranten Syndroms. Es wird die Bedeutung der Balance zwischen cholinergem und adrenergem Neurotransmittersystem darstellt dächtnis, sondern auch Aufmerksamkeit, Vigilanz und den Schlaf-Wach-Rhythmus. Der pathophysiologische Hintergrund ist der, dass die cholinergen Neurone des basalen Vorderhirns der am weitesten rostral gelegene Teil des aufsteigenden aktivierenden retikulären Systems (ARAS) und damit die strukturelle Basis der neokortikalen Aktivierung betroffen sind [1].

\section{Delirien und hirnorganische Störungen bei somatischen Erkran- kungen und deren Behandlung}

Damit ist der Bogen zu den deliranten Syndromen geschlagen. Definitionsgemäß unterscheidet sich das Delir von der Demenz vor allen Dingen durch eine Störung von Aufmerksamkeit und Wachheit. Zudem tritt es oft relativ akut und mitunter auch nur vorübergehend auf; deshalb sind Zeitkriterien weitere Unterscheidungsmerkmale. Neben Wahrnehmungsstörungen finden sich affektive Störungen und solche der Psychomotorik. Systematische Untersuchungen zur Häufigkeit und zum Verlauf finden sich bis jetzt wenig. Aus Untersuchungen in Allgemeinkrankenhäusern finden sich jedoch Daten, dass bis zu einem Drittel der Patienten bei Aufnahme und im Verlauf ein Delir entwickeln. Die Raten sind in operativen Fächern oft noch höher, insbesondere in der Orthopädie und Kardiochirurgie (20-50\%). Bekanntermaßen geht ein Delir mit einem erhöhten Komplikationsrisiko für Stürze und Pneu- monien einher. Auch kommt es oft nicht zu einer vollständigen Restitution. Ein erhöhtes Risiko besteht bei höherem Alter, vorbestehender Demenz, Seh- und Hörstörungen, einem reduzierten Allgemeinzustand, metabolischen Störungen und Infektionen. Polypharmazie oder auch Medikamentenabhängigkeit sind weitere - oft miteinander zusammenhängende - Risikofaktoren [23]. - Abbildung 2 fasst schematisch die Neurobiologie des deliranten Syndromes zusammen. Aus diesem Modell lässt sich auch die bekannte hirnleistungsmindernde Wirkung von allen Pharmaka mit anticholinergen Nebenwirkungen ableiten. Dies sind durchaus nicht nur Psychopharmaka, sondern gerade im Alter häufig verordnete Antiarrhythmika, Antihistaminika oder Mittel gegen Miktionsstörungen.

\section{Depressionen}

Depressionen sind mit einer Häufigkeit bis zu 20\% die häufigste psychische Erkrankung im Alter und treten sehr oft zusammen mit körperlichen Erkrankungen auf [23]. Unter dem Aspekt der Verhandlungsfähigkeit müssen hier 2 Aspekte besonders berücksichtigt werden. Zum einen sind bei Depressionen sehr häufig (nicht nur vorübergehend) kognitive Störungen vorhanden, die sich in einer Verlangsamung des Denkens, Konzentrations- und Aufmerksamkeitsstörungen zeigen. Relevant sind v. a. auch qualitative Veränderungen des
Denkens, wie sie typischerweise zu einer Depression gehören. In den letzten Jahren wurden Untersuchungen veröffentlicht, die zeigten, dass insbesondere Entscheidungen für bzw. gegen lebenserhaltende Therapien durch das Vorhandensein einer Depression beeinflusst wurden, und dass Depressive auch ein zunehmendes Interesse an Sterbehilfe zeigten $[6,12,21]$. Neben der Depressivität hingen diese Einstellungen bzw. Entscheidungen auch vom allgemeinen Gesundheitszustand und z. B. von der religiösen Orientierung ab. Innerhalb des depressiven Syndroms waren offensichtlich die Hoffnungslosigkeit und auch die Suizidalität relevante Parameter für diese Entscheidungsfindung. Dass somit ein depressiver Patient in einer Verhandlung - auch wenn er nicht wahnhaft ist - seine Interessen nur eingeschränkt adäquat vertreten kann, liegt auf der Hand.

\section{Strukturierte Erhebungs- methoden zur Beurteilung von Kompetenz}

In Anbetracht fehlender Operationalisierungen wird häufig ein kategorisches Expertenurteil im Sinne eines „Goldstandards" verwendet. Diese weit verbreitete Methode zeigt jedoch eine geringe Interraterreliabilität. Demgegenüber hätte die Anwendung strukturierter Verfahren, z. B. psychometrischer Tests, den Vorteil, vergleichbare Daten und somit eine hohe Reliabilität $\mathrm{zu}$ bringen. Häufig sind jedoch 
diese Ergebnisse allein ethisch bzw. juristisch nicht relevant. Neuropsychologisch spielen für die Kompetenz bzw. Verhandlungsfähigkeit neben Gedächtnisleistungen v. a. die Exekutivfunktionen eine große Rolle [4, 7, 8, 22]. Standardinstrumente zu ihrer Messung sind z. B. der Trail-making-Test B [20] oder der Wisconsin-cardsorting-Test [4]. Interessant wäre es nun, wenn man beide methodischen Vorgehensweisen miteinander kombinieren bzw. vergleichen würde. Dies wurde z. B. in einer Untersuchung [18] bei 70 Patienten mit einer Alzheimer-Erkrankung gemacht. Ein dichotomes Expertenurteil wurde mit einer Reihe etablierter neuropsychologischer Tests und mit so genannten Demenzskalen verglichen. Die höchste Korrelation zeigte sich interessanterweise zu letztgenannten Skalen, insbesondere zum „minimental status test" (MMSE) [11] oder der "global deterioration scale“ (GDS) [19].

Im englischen Schrifttum findet sich eine Reihe von strukturierten Interviews zum Kompetenzassessment (z. B. „Hopkins competency assessment test“, „capacity assessment tool“, „capacity to consent to treatment instrument"), die z. T. auch auf bestimmte Situationen ausgerichtet sind, z. B. der Kompetenz zur Regelung finanzieller Angelegenheiten (z. B. [22]). Die Arbeitsgruppe um Marson publizierte im Jahr 2000 eine operationalisierte Definition von "legal standards“, wie sie in • Tabelle 1 dargestellt sind [15].

Von all diesen Methoden ist sicherlich der oben schon erwähnte MMSE bisher am besten untersucht [13]. Er wurde ursprünglich als Screeningtest entwickelt, um Verdachtsfälle einer Demenz zu identifizieren. Inzwischen wird er vielfach auch zur Schweregradbestimmung von Demenzen eingesetzt, z. B. auch zur Definition der Einschlusskriterien in Arzneimittelzulassungsstudien. Weil er einfach anzuwenden ist, ist er auch durchaus für die Fragestellung der Verhandlungsfähigkeit interessant. Nach verschiedenen Untersuchungen konnte gezeigt werden, dass bei Werten unter 16 sicher von einer Störung der Kompetenz auszugehen ist, bei Werten über 26 allenfalls fraglich. Somit sind Werte in dem Bereich dazwischen überprüfungsbedürftig und natürlich auch von der untersuchten Population abhängig $[9,10,14,16,17]$. In einer Metaanalyse
Rechtsmedizin $2005 \cdot 15: 143-147$

DOI 10.1007/s00194-005-0318-2

c) Springer Medizin Verlag 2005

G. Stoppe

\section{Die Verhandlungsfähigkeit des alten (multimorbiden) Patienten}

\section{Zusammenfassung}

Die Anforderungen an die Verhandlungsfähigkeit entsprechen im Wesentlichen dem „Kompetenzmodell“ in der neuropsychiatrischen Literatur: Eine Person muss einen Sachverhalt verstehen, in einen $\mathrm{Zu}$ sammenhang einordnen, die Konsequenzen bedenken sowie eine Entscheidung fällen und ausdrücken können. Gesundheitliche Störungen, die die Verhandlungsfähigkeit beeinflussen, sind im Alter vorwiegend bei Demenzen, Depressionen und/ oder Delirien zu erwarten. Relevante Fakten zu diesen Syndromen werden dargestellt. Um die Interraterreliabilität und die Validität von Kompetenzbeurteilungen zu optimieren, erscheint es sinnvoll, strukturierte „assessments" unter Einbeziehung auch psychometrischer Tests heranzuziehen, statt sich allein auf ein klinisches Glo-

bal- oder Gesamturteil zu verlassen. Bestuntersucht ist bis heute der "Mini-Mental Status Test" (MMSE). Bei Werten von weniger als 16 Punkten ist danach sicher von einer Kompetenzstörung auszugehen, bei solchen über 26 allenfalls fraglich. Skalen und Instrumente, die Depressivität oder andere psychopathologische Funktionen quantifizieren, wurden hierzu bisher nicht untersucht. Mit diesem Thema befasste Juristen sollten eine Basiskompetenz im Umgang mit diesen orientierenden Instrumenten erwerben.

\section{Schlüsselwörter}

Verhandlungsfähigkeit .

Kompetenzmodell · Alte Patienten .

Mini-mental status test · Demenz ·

Delirium · Depressionen

\section{Competency of the (multimorbid) aged patient}

\section{Abstract}

The requirements for a person to take part in legal proceedings are defined in the competency model of the neuropsychiatric scientific literature. Accordingly a person must be able to understand, appreciate and reason a problem and decide and express an opinion. In the elderly, conditions which have an impact on competency are mainly dementia, delirium and depression and relevant details of these disorders are described. Studies revealed that the best results for interrater reliability and validity of competency assessments, were obtained when structured assessments and neuropsychological tests were combined with clinical global as- sessment. The mini mental status examination (MMSE) is the best tested method to date. Values below 16 are clearly indicative of reduced competency, and over 26 are mostly not. Considering the frequency of dementia and depression and the aging population, it might be useful for legal professionals to have some knowledge of these screening tests.

\section{Keywords}

Competency to participate in proceedings . Competence model $\cdot$ Aged patient . Mini-mental status test - Delirium . Dementia. Depression 


\section{Hier steht eine Anzeige.}

Springer

kamen Kim et al. zu dem Schluss, dass gegenwärtig Beurteilungen der Kompetenz von Individuen ,in relativer Isolation“ gemacht werden, indem sie intuitiv relativ breite Kriterien zum Einsatz bringen [13]. Eine Methode, die auf einem operationalisierten Expertenurteil, u. U. unter Einbeziehung einfacher Skalen und Instrumente beruht, hätte das Potenzial, Schwellenwerte für die Kompetenz transparent und zuverlässig zu machen. Dies wäre ein extrem sinnvoller Beitrag der empirischen Wissenschaft zur ethischen Praxis.

\section{Fazit für die Praxis}

Aufgrund der vorliegenden Überlegungen und empirischen Untersuchungen ist zu wünschen, dass die Beurteilung der Verhandlungsfähigkeit alter und v. a. auch multimorbider Patienten die derzeit schon vorhandenen Möglichkeiten nutzt. Danach sollte jede Begutachtung Auskunft über die Anknüpfungstatsachen, die verwendeten Tests und Methoden sowie deren Befunde geben. In Anbetracht der Häufigkeit von Depressionen, Demen-

Tabelle 1

Operationalisierung der "legal standards" zum Kompetenzassessment. (Marson et al. [15])

Operational definitions of legal standards

Legal - Evidencing a treatment choice for or against treatment

Standard 1 - Focuses not on the quality of the patient's decision but on the presence or absence of a decision

Legal Making the reasonable treatment choice

Standard 2 - The capacity to reach the reasonable, the right or the responsible choice. The emphasis is on outcome rather than on the mere fact of decision or how it has been reached....

\begin{tabular}{|c|c|}
\hline $\begin{array}{l}\text { Legal } \\
\text { Standard } 3\end{array}$ & $\begin{array}{l}\text { - Appreciating consequences of a treatment choice } \\
\text { - The capacity to realize the implications of a treatment choice in terms of its } \\
\text { emotional impact, rational requirements, and future consequences }\end{array}$ \\
\hline $\begin{array}{l}\text { Legal } \\
\text { Standard } 4\end{array}$ & $\begin{array}{l}\text { - Providing rational reasons for a treatment choice } \\
\text { - The ability to use logical processes to compare the benefits and risk of various } \\
\text { treatment options and weigh this information to reach a decision }\end{array}$ \\
\hline Legal & - Understanding the treatment situation \\
\hline Standard 5 & $\begin{array}{l}\text {-A memory for words, phrases, ideas.....and beyond mere reception, storage } \\
\text { and retrieval of such information, the patient must be able to comprehend } \\
\text { the fundamental meaning of information about treatment }\end{array}$ \\
\hline
\end{tabular}

zen und Delirien sollte bei körperlich kranken und alten Menschen stets das Vorliegen einer solchen Störung überprüft bzw. ausgeschlossen werden. Auch sollte dies dokumentiert werden.

Hierzu kann auf etablierte Suchtests (z. B. MMSE [11], GDS [26]), neuropsychologische Tests und strukturierte Assessments (geriatrische Assessments, Kompetenzassessments) zurückgegriffen werden. Es hat sich gezeigt, dass Suchtests sehr wohl auch von hierfür trainiertem Praxispersonal kompetent durchgeführt werden können. Es könnte somit ein pragmatisches Vorgehen sein, wenn Juristen, die die Verhandlungsfähigkeit beurteilen sollen, oder auch Notare, sich Kompetenz in Umgang und Einsatz dieser Screeninginstrumente verschaffen. Sie sind gleichzeitig eine gute Dokumentation von Befunden. Verdachtsfälle könnten dann dem sachverständigen Psychiater zur Beurteilung zugewiesen werden.

\section{Korrespondierender Autor}

\section{Prof. Dr. G. Stoppe}

Universitäre Psychiatrische Kliniken, Wilhelm Klein-Strasse 27, CH-4025 Basel, Schweiz

E-Mail: gabriela.stoppe@upkbs.ch

Interessenkonflikt: Der korrespondierende Autor versichert, dass keine Verbindungen mit einer Firma, deren Produkt in dem Artikel genannt ist, oder einer Firma, die ein Konkurrenzprodukt vertreibt, bestehen.

\section{Literatur}

1. Ahrendt T (1999) Pathologische Anatomie der Alzheimer Krankheit. In: Förstl H, Bickel H, Kurz A (Hrsg) Alzheimer Demenz. Grundlagen, Klinik und Therapie. Springer, Berlin Heidelberg New York, S 87-106

2. American Psychiatric Association (APA) (1994) Diagnostic and statistical manual of mental disorders, 4th edn. American Psychiatric Association, Washington/DC. Deutsche Übersetzung und Bearbeitung: Diagnostisches und statistisches Manual psychischer Störungen DSM-IV (1996). Hogrefe, Göttingen

3. Appelbaum PS, Grisso T (1988) Assessing patients capacities to consent to treatment. N Engl J Med 319:1635-1638

4. Baddeley AD (1996) Exploring the central executive. Q J Exp Psychol 49A:5-28

5. Bickel H (1999) Epidemiologie der Demenzen. In: Förstl H, Bickel H, Kurz A (Hrsg) Alzheimer Demenz. Grundlagen, Klinik und Therapie. Springer, Berlin Heidelberg New York, , S 9-32

6. Blank K, Robison J, Doherty E, Prigerson H, Duffy J, Schwartz HI (2001) Life-sustaining treatment and assisted death choices in depressed older patients. J Am Geriatr Soc 49:153-161 
7. Dymek MP, Atchison P, Harrell L, Marson DC (2001) Competency to consent to medical treatment in cognitively impaired patients with Parkinson's disease. Neurology 56:17-24

8. Earnst KS, Wadley VG, Aldridge TM, Boothe Steenwyk A, Hammond AE, Harrell LE, Marson DC (2001) Loss of financial capacity in Alzheimer's disease: the role of working memory. Aging Neuropsychol Cogn 8:109-119

9. Etchells E, Darzins P, Silberfeld M et al. (1999) Assessment of patient capacity to consent to treatment. J Gen Intern Med 14:27-34

10. Fitten LJ, Lusky R, Hamann C (1990) Assessing treatment decision making capacity in elderly nursing home residents. J Am Geriatr Soc 38:1097-1104

11. Folstein MF, Folstein SE, McHugh PR (1975) „'MiniMental State". A practical method for grading the cognitive state of patients for the clinician. J Psychiatry Res 12:189-198

12. Ganzini L, Lee MA, Heintz RT, Bloom JD, Fenn DS (1994) The effect of depression treatment on elderly patients' preferences for life-sustaining medical therapy. Am J Psychiatry 151:1631-1636

13. Kim SYH, Karlawish JHT, Caine ED (2002) Current state of research on decision-making competence of cognitively impaired elderly persons. Am J Geriatr Psychiatry 10:151-165

14. Krynski MD, Tymchuk AJ, Ouslander JG (1994) How informed can consent be? New light on comprehension among elderly people making decisions about enteral tube feeding. Gerontologist 34:36-43

15. Marson DC, Earnst KS, Jamil F, Bartolucci A, Harrell LE (2000) Consistency of physicians' legal standard and personal judgements of competency in patients with Alzheimer's disease. J Am Geriatr Soc 48:911-918

16. Molloy DW, Silberfeld M, Darzins P et al. (1996) Measuring capacity to complete an advanced directive. J Am Geriatr Soc 44:660-664

17. Pruchno RA, Smyer MA, Rose MS, Hartman-Stein PE, Henderson-Laribee DL (1995) Competence of long term care residents to participate in decisions about their medical care: a brief, objective assessment. Gerontologist 35:622-629

18. Pucci E, Belardinelli N, Borsetti G, Rodriguez D, Signorino $M$ (2001) Information and competency for consent to pharmacologic clinical trials in Alzheimer disease: an empirical analysis in patients and family caregivers. Alzheimer Dis Assoc Disord 15:146-154

19. Reisberg B, Ferris SH, Leon M de, CrookT (1982) The global deterioration scale for assessement of primary degenerative dementia. Am J Psychiatry 139:1136-1139

20. Reitan RM (1958) Validity of the trail making test as an indication of organic brain damage. Percept Mot Skills 9:127-130

21. Rosenblatt L, Block SD (2001) Depression, decision making, and the cessation of life-sustaining treatment. West J Med 175:320-325

22. Spear Bassett S (1999) Attention: neuropsychological predictor of competency in Alzheimer's disease. J Geriatr Psychiatry Neurol 12:200-205

23. Stoppe $G$ (2000) Die kardinalen psychiatrischen Probleme im Alter. Internist 41:538-543

24. Stoppe G (2002) Diagnose und Differentialdiagnose der Demenzerkrankungen. In: Wächtler C (Hrsg) Demenzen. Thieme, Stuttgart, S 24-50

25. World Health Organization (WHO) (1991) Tenth revision of the international classification of diseases, Chapter V (F): mental and behavioural disorders (including disorders of psychological development). Clinical Descriptions and Diagnostic guidelines. WHO, Genf

26. Yesavage JA, BrinkTL, Rose TL (1983) Development and validation of a geriatric depression screening scale: a preliminary report. J Psychiatry Res 17:37-49

\section{Madea, B., Mußhoff F. (Hrsg.) \\ Haaranalytik, Technik und Inter- pretation in Medizin und Recht}

Deutscher Ärzteverlag 2003, 395 S., 125 Abb., 66 Tab., (ISBN 3-7691-0437-4), 89.95 EUR

Die bereits sehr alte Methode der Haaranalytik hat in den letzten Jahrzehnten bezüglich ihrer Anwendbarkeit und Aussagekraft erhebliche Erweiterungen erfahren und ist deshalb für zahlreiche forensische, kriminologische und klinische Fragestellungen heute außerordentlich relevant. So findet sie Anwendung sowohl beim Nachweis illegal konsumierter Betäubungsmittel und krimineller Vergiftungen als auch ergänzend für die Schuldfähigkeitsbeurteilung. Die Haaranalytik hat außerdem im Rahmen von Dopingkontrollen einen bedeutenden Stellenwert. Weiterhin erlangt sie zunehmende Relevanz in Fragen der Verkehrs- und Berufseignung. Klinisch relevante Anwendungsgebiete sind unter anderem der Nachweis einer chronischen Exposition mit Giften am Arbeitsplatz oder die Prüfung von Abstinenz bzw. Compliance eines Patienten bei Medikamenteneinnahme. Zusätzlich können die strukturelle und die DNA-Analyse an Haaren wichtige Beiträge bei spurenkundlichen Identifizierungen leisten.

All diese Anwendungsgebiete der Haaranalytik werden im vorliegenden Buch übersichtlich behandelt. Dabei sind die verschiedenen zur Anwendung kommenden Methoden verständlich erklärt und ausführlich beschrieben, ergänzt durch ein umfangreiches und aktuelles Literaturverzeichnis sowie einen gesonderten Abschnitt über Qualitätssicherung bei forensisch-toxikologischen Untersuchungen von Haarproben und bei der Analyse von STRs. Die biologischen Grundlagen des Einbaus von Fremdsubstanzen in die Haare sind vorab dargelegt und erleichtern das Verständnis.

Ausführlich werden die rechtlichen Grundlagen und Folgen der Haaranalytik abgehandelt, wobei Grenzen der Methode bzw. ihrer Anwendbarkeit und Interpretation angemessen kritisch aufgezeigt werden. Es werden hier auch aktuelle Probleme wie beispielsweise die Einführung der
Generellen Durchführung eines „,Workplace Drug Testing" diskutiert.

Sehr informativ sind der kurze historische Überblick über die Anfänge der Haaranalytik und ihre Entwicklung bis in die moderne Zeit sowie zahlreiche interessante und teilweise bekannt gewordene Fallbeispiele, bei denen die Haaranalytik zur Aufklärung beitragen konnte. Insgesamt werden die Kapitel immer wieder durch Fallbeispiele und praxisbezogene Fragestellungen aufgelockert, so dass das Buch trotz der methodisch anspruchsvollen Beiträge angenehm zu lesen ist.

In seiner Vollständigkeit und gleichzeitigen Übersichtlichkeit und Praxisnähe schließt dieses Standardwerk eine Lücke in der deutschsprachigen Literatur über Haaranalytik.

E. Türk (Hamburg) 